\title{
Overview of a cyber-enabled wireless monitoring system for the protection and management of critical infrastructure systems
}

\author{
Jerome P. Lynch ${ }^{* a b}$, Vineet Kamat ${ }^{\mathrm{a}}$, Victor C. Li ${ }^{\mathrm{a}}$, Michael Flynn ${ }^{\mathrm{b}}$, Dennis Sylvester ${ }^{\mathrm{b}}$, \\ Khalil Najafi ${ }^{\mathrm{b}}$, Timothy Gorden ${ }^{\mathrm{c}}$, Michael Lepech ${ }^{\mathrm{d}}$, Abbas Emami-Naeini ${ }^{\mathrm{e}}$, \\ Alex Krimotat ${ }^{\mathrm{e}}$, Mohammed Ettouney ${ }^{\mathrm{f}}$, Sharada Alampalli ${ }^{\mathrm{g}}$, Tayfun Ozdemir ${ }^{\mathrm{h}}$ \\ ${ }^{\mathrm{a}}$ Dept. of Civil and Environmental Engineering, University of Michigan, Ann Arbor, Michigan; \\ ${ }^{b}$ Dept. of Electrical Eng. and Computer Science, University of Michigan, Ann Arbor, Michigan; \\ ${ }^{c}$ University of Michigan Transportation Research Institute, Ann Arbor, Michigan; \\ ${ }^{\mathrm{d}}$ LFL Associates, Ann Arbor, Michigan; \\ ${ }^{\mathrm{e}} \mathrm{SC}$ Solutions, Santa Clara, California; \\ ${ }^{\mathrm{f}}$ Weidlinger Associates, New York, New York; \\ ${ }^{g}$ Prospect Solutions, Loudonville, New York ; \\ ${ }^{\mathrm{h}}$ Monarch Antenna, Ann Arbor, Michigan;
}

\begin{abstract}
The long-term deterioration of large-scale infrastructure systems is a critical national problem that if left unchecked, could lead to catastrophes similar in magnitude to the collapse of the I-35W Bridge. Fortunately, the past decade has witnessed the emergence of a variety of sensing technologies from many engineering disciplines including from the civil, mechanical and electrical engineering fields. This paper provides a detailed overview of an emerging set of sensor technologies that can be effectively used for health management of large-scale infrastructure systems. In particular, the novel sensing technologies are integrated to offer a comprehensive monitoring system that fundamentally addresses the limitations associated with current monitoring systems (for example, indirect damage sensing, cost, data inundation and lack of decision making tools). Self-sensing materials are proposed for distributed, direct sensing of specific damage events common to civil structures such as cracking and corrosion. Data from self-sensing materials, as well as from more traditional sensors, are collected using ultra low-power wireless sensors powered by a variety of power harvesting devices fabricated using microelectromechanical systems (MEMS). Data collected by the wireless sensors is then seamlessly streamed across the internet and integrated with a database upon which finite element models can be autonomously updated. Life-cycle and damage detection analyses using sensor and processed data are streamed into a decision toolbox which will aid infrastructure owners in their decision making.
\end{abstract}

Keywords: wireless sensors, self-sensing materials, cyber-infrastructure, structural health monitoring, damage detection

\section{INTRODUCTION}

Civil infrastructure such as bridges, roadways, and water pipelines, are critical to providing mobility, clean water, and other elements essential to every citizen's quality of life. In fact, civil infrastructure in the U.S. has performed so well that they are often "taken for granted" until a catastrophic failure occurs, as witnessed by the collapse of the I-35W Bridge in Minnesota in 2007. Perhaps more unsettling is that the I-35W Bridge is not as rare of an event as the public may realize. A recent study reports the partial and total collapse of over 130 bridges in the United States from 1989 to 2000 [1]. Sources of failure were cited to be from poor maintenance, flaws introduced during design and construction, and from triggering events such as impact loading, scour and earthquakes. Aside from catastrophic collapse, deterioration is another serious concern; the American Society of Civil Engineers (ASCE) estimates that over \$1.6 trillion are necessary over the next five years to adequately maintain the national infrastructure inventory. In the case of bridges, the Federal Highway Administration (FHWA) reported that $25 \%$ of the national bridge inventory was

*jerlynch@umich.edu; phone: (734) 615-5290; fax: (734) 764-4292; http://www-personal.umich.edu/ jerlynch/ 
structurally deficient, the same classification given to the I-35W Bridge a few years before failure. To compound the problem, the economic resources that are available to maintain our infrastructure are shrinking at an alarming rate. For example, the Highway Trust Fund will have a several billion dollar deficit by year's end [2].

A contributing factor to the problem is that current approaches to inspecting civil infrastructure fall short in providing a complete picture of the condition of a structure. Specifically, infrastructure owners are still reliant on the use of visual inspections to determine operational conditions and to devise follow-up maintenance actions. For example, the National Bridge Inspection Program (NBIP) administered by the FHWA mandates bi-annual inspection of every highway bridge in the United States [3]. Unfortunately, the visual inspections mandated by the NBIP are known to be subjective, thereby hindering reliable structural management [4]. While nondestructive evaluation (NDE) technologies have begun to be explored for field use, their application remains rare because of high costs and the need for trained technicians to operate NDE equipment. Recently, infrastructure owners have begun to realize the need for continuous and autonomous monitoring of their assets through the use of permanent monitoring systems. Termed structural health monitoring (SHM) systems, such systems are designed to generate objective measurements that aid in cost-effective infrastructure management.

While great technological advances have been made in recent years on many fronts in the SHM field, there still remain very few implementations of true SHM systems in actual operational structures. Factors often cited for the lack of widespread adoption are many:

- Indirect damage sensing: generally speaking, sensors in common use do not detect damage directly [5]. Rather, sensors make measurement of structural responses with physics-based models necessary to correlate measurements to damage states. Given the many complexities inherent to this inverse problem, robust algorithms that can be applied in a generic (e.g., black-box) manner are limited [6, 7].

- Point-based sensing: widely used sensors, such as strain gages and accelerometers, are point-based sensors. To accurately identify damage which can be spatially distributed (e.g., cracking or corrosion), a dense network of point-based sensors are necessary to generate input data for analytical models that extrapolate point measurements to predict the behavior of a component or structure.

- Tethered sensors: for decades, monitoring systems have been tethered. Unfortunately, the wiring demands for large infrastructure systems are high (often kilometers of wires) leading to expensive and time-consuming installation [8]. While wireless sensors have emerged as a promising alternative [9], the absence of power sources offering decades of life expectancy limit their adoption.

- Lack of system scalability: while hundreds of sensors can be installed in a single structure, management of the data created remains an unresolved problem. Current data management methods are overly reliant on manual processing; the result has been an order of magnitude less data processed than that generated. Hence, transient damage signatures can go undetected due to sporadic investigation of the data streams.

- Incompatibility between data and decision-making: no matter how sophisticated the monitoring system is, it is only of real use if it provides end-users with information amenable to the decision making process. A glaring gap currently exists between sensed data and the information infrastructure owners critically need to confidently make informed and optimal decisions regarding maintenance and upkeep. Until this chasm is bridged, the utility of structural monitoring will remain well below its enormous potential.

This paper presents the planned efforts of a multidisciplinary engineering team assembled to create a next-generation structural health monitoring system for the protection of large-scale infrastructure systems (e.g., bridges, roadways, pipelines, buildings). The architectural design of the proposed structural health monitoring system fundamentally addresses the aforementioned limitations (indirect point-based sensors, high system costs due to tethered sensing, data inundation, and the chasm between sensed data and decision making). The paper first provides an overview of the architectural design of the system followed by a detailed description of key system components.

\section{ARCHITECTURAL OVERVIEW OF THE PROPOSED SYSTEM}

A structural health monitoring architecture is proposed for the management of critical infrastructure systems. As shown in Fig. 1, the monitoring system architecture is delineated along three length-scales spanning from the individual sensor (i.e., component scale) up to the decision making process that incorporates processed data from bridge fleets (i.e., 


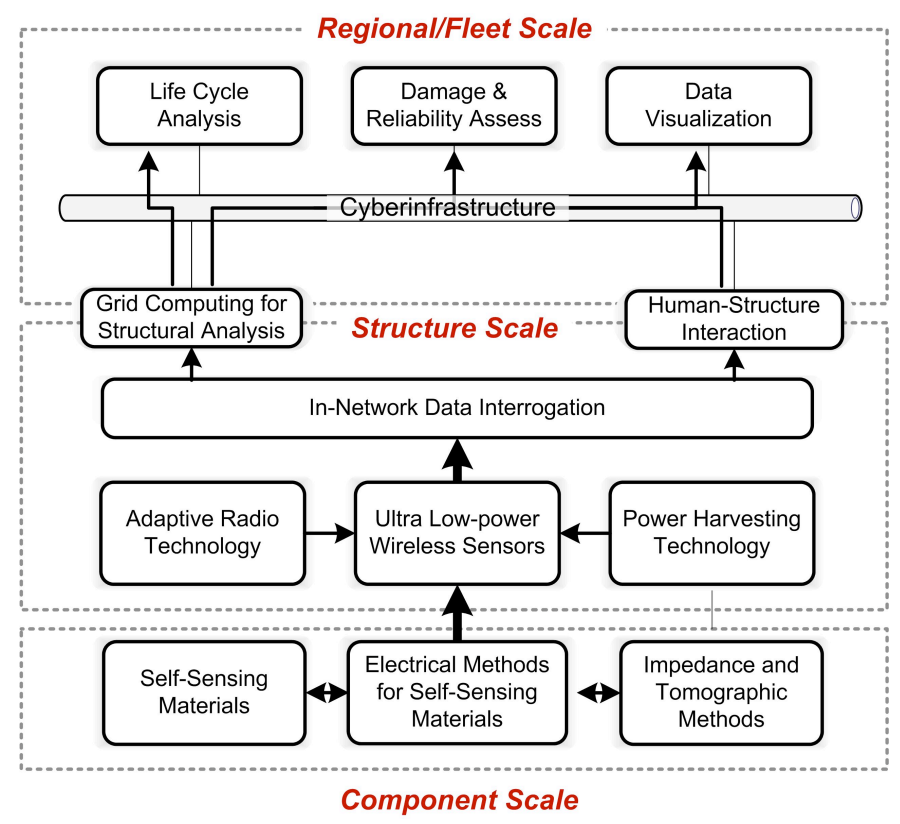

Fig. 1. Overview of the cyber-enabled wireless structural health monitoring system for infrastructure systems.

regional scale). A variety of innovative sensor technologies are proposed at the component-scale to improve the type of sensed data available for damage detection analysis. While traditional point-sensors (e.g., strain gages, accelerometers) can still be used within the proposed monitoring system, the system emphasizes the use of self-sensing materials. Selfsensing materials are ones that have a sensing transduction mechanism embedded; in other words, self-sensing materials exhibit changes in their electrical properties to mechanical (e.g., strain) or chemical (e.g., corrosion) stimulus. Selfsensing materials are advantageous because they are capable of making a measurement wherever the material is located; this opens many opportunities for spatial mapping of structural behavior.

To collect data from self-sensing construction materials, as well as from traditional sensors, a low-cost wireless sensor network is proposed at the structure-scale. Wireless sensors are an enabling technology because they facilitate dense instrumentation of large civil structures. While wireless sensors have recently proven themselves as viable alternatives to wired sensors, some technological bottlenecks still exist. In the proposed system, two specific bottlenecks are addressed: power and communication reliability. To address the power challenge, an ultra low-power wireless sensor node will be proposed based on $0.18 \mu \mathrm{m} \mathrm{CMOS} \mathrm{technology.} \mathrm{The} \mathrm{resulting} \mathrm{sensor} \mathrm{will} \mathrm{enjoy} \mathrm{a} \mathrm{power} \mathrm{draw} \mathrm{less} \mathrm{than} 20$ $\mathrm{mW}$ which is an order of magnitude less than current wireless sensors (which draw 100's of $\mathrm{mW}$ ). Integrated with this impressively low-power node, are vibration and electromagnetic (EM) power harvesting components fabricated in silicon using MEMS processes. Wireless communication reliability is also proposed based on adaptive, self-structuring antenna technology where the antenna beam can be focused and steered.

At the regional scale, cyberinfrastructure (i.e., internet-based information technologies) bridges the gap between wireless sensors installed in a structure and an array of software services remotely located on the internet. The architectural design of the monitoring system is intended to have data flow upward from the sensors to the sensor network and on to the internet where the data is assimilated with analytical models for damage detection, risk and reliability analysis and life-cycle analysis. Another powerful facet of the cyberinfrastructure architecture is the opportunity to provide on-site inspectors powerful user interfaces for interaction with the pervasive sensor network. A key novelty of the proposed system is that the flow of data in the monitoring system is designed to feed into an integrated decision-making tool that would bridge the gap between sensing and optimal decision making. This tool would allow owners to attain adequate and safe infrastructure performance at reasonable upkeep costs. The decision making tools integrated in a "Decision Making Toolbox" include reliability and risk assessment, cost-benefit analysis and life-cycle costing tools. Thresholds regarding acceptance limits and warning signs will be incorporated in the decision analysis algorithm to provide realtime assessment of unsafe infrastructure elements. 


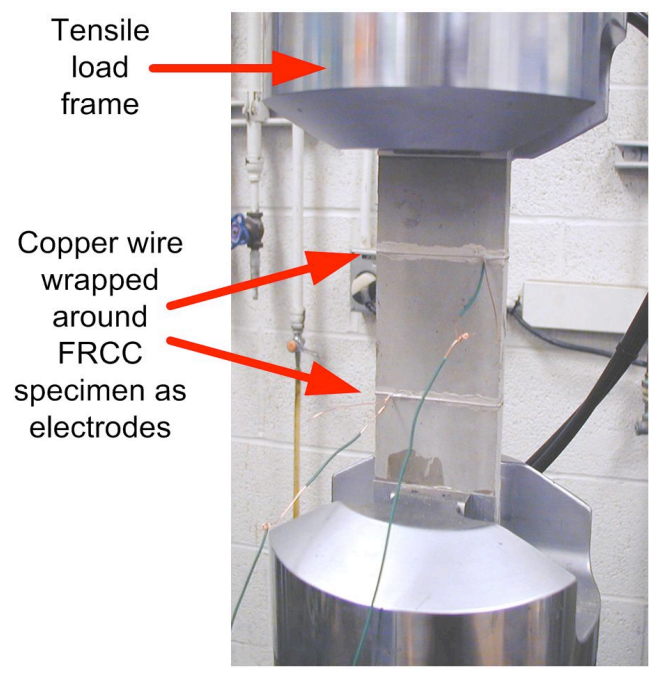

(a)
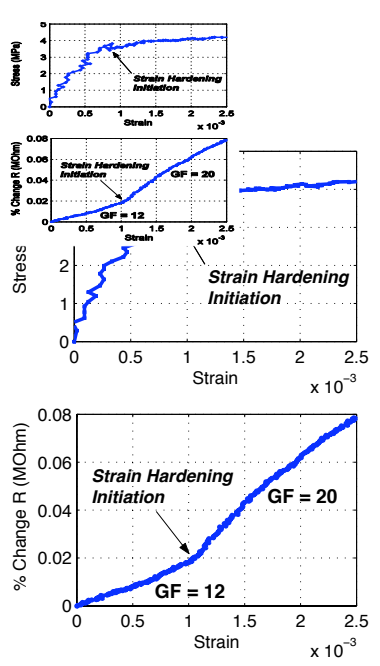

(b)

Fig. 2. (a) ECC plate element loaded in monotonic axial tension as resistivity is measured by two-point probing and (b) resistivity versus strain plots.

The advances proposed at each length scale are not in isolation of one another; rather, the approach to be taken occurs in a coordinated and interconnected manner across the entire system architecture to yield a paradigm shift in how infrastructure systems are monitored. Furthermore, the monitoring system proposed is sufficiently general that it can be applied to any civil infrastructure system including roads, pipelines, tunnels and bridges. However, the structural health monitoring system proposed herein will be demonstrated initially on bridges.

\section{HIGHLIGHT OF SYSTEM COMPONENTS}

\subsection{Self-sensing materials for distributed sensing}

In lieu of reliance on point-sensors, a radical new approach to sensing is offered based on multifunctional materials. Multifunctional materials are designed to carry-out more than one function (e.g., mechanical support). In this project, a new self-sensing cementitious material termed Engineered Cementitious Composites (ECC) is explored for the construction of future infrastructure systems. Mechanically, ECC is a fiber reinforced cementitious composite designed to exhibit high ductility under tensile loading (e.g., tensile strain capacities between 3 and 8\%) [10]. The tensile strength of ECC can be as high as $10 \mathrm{MPa}$, with compressive strengths on the same order as high-strength concrete. However, the electrical conductivity of ECC are of principal interest in this project because changes in conductivity can be correlated to strain and crack damage [11]. As shown in Fig. 2a, the resistivity (inverse of conductivity) of an ECC plate using polymeric and steel fibers is measured as tensile loading is applied. Stress-strain curves for the two ECC plates reveal ductile strain hardening while the resistivity curves (Fig. 2b) illustrate linear variations in resistivity with applied strain. While the piezoresistive properties of ECC can be potentially used to measure the material strain response, electromechanical sensing methods have encountered some implementation issues due to environmental factors such as temperature and relative humidity inducing conductivity changes of their own. Environmental influences on the piezoresistive behavior of ECC will be a core focus of the proposed study.

To employ self-sensing ECC as a distributed sensor capable of mapping crack states, imaging techniques commonplace to the biomedical field will be adopted. Specifically, electrical impedance tomography (EIT) is adopted to map the conductivity of ECC structural specimens [12]. The goal is the visualization of material conductivity in two- or threedimensions based upon voltage measurements collected in ECC specimens in a multitude of probe locations. Conductivity reconstruction is a difficult nonlinear inverse problem defined by an underdetermined set of linear equations based on Poisson equation:

$$
\nabla[\sigma(x, y, z) \nabla \phi(x, y, z)]=0
$$



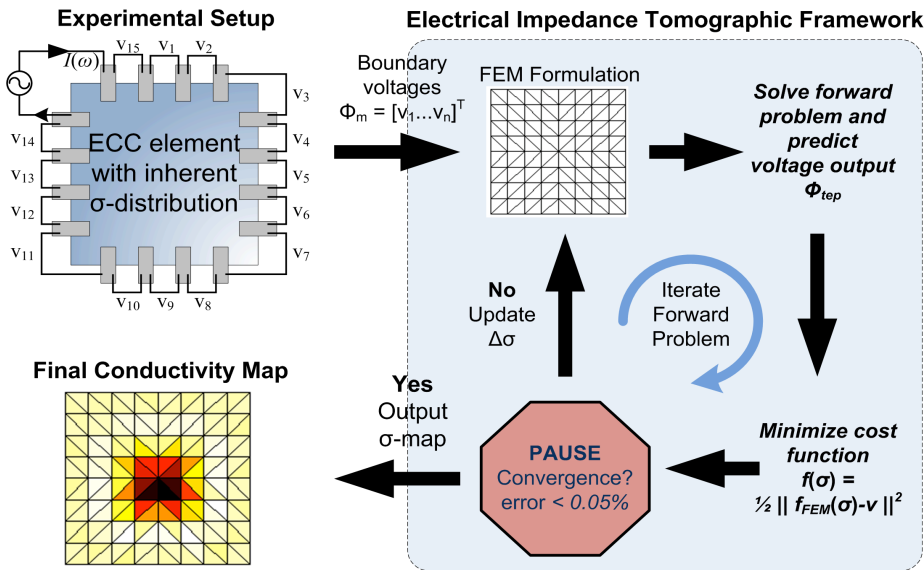

Fig. 3. Change in current flow map as a result of crack damage in ECC elements.

where $\sigma$ is the material conductivity and $\phi$ is the electric potential at coordinates $x, y$ and $z$. Solutions of the inverse problem (i.e., determining $\sigma$ based on boundary voltage measurements, $v$ resulting from an electrical stimulus on the boundary) exist [13]. Finite element models (FEM) that describe the forward problem (Poisson equation) will be implemented. The inverse solution will be solved using regularized least square analysis (e.g., Tikhonov regularization). The end result of EIT analysis is a conductivity map in which cracks (i.e., zero-conductivity voids) can be identified reliably. An overview of the EIT approach to distributed sensing of ECC specimens is shown in Fig. 3.

\subsection{Ultra low-power wireless sensor node}

Wiring large-scale civil structures (most especially long-span bridges) with a wired structural monitoring system can be a difficult and labor-intensive task [8]. These challenges have historically driven the cost of the monitoring system high, in many cases beyond the fiscal means of bridge owners. Even when conditions justify the installation of a monitoring system, low sensor densities are adopted to keep the cost of the system as low as possible. Since deterioration is inherently a localized phenomenon, low densities are insufficiently scaled for the accurate detection of damage [14]. In the mid 1990's, wireless sensors were proposed as an alternative approach to monitoring civil structures at a substantially lower cost [8]. Since that time, a large number of wireless sensor prototypes have been proposed with many even having been proven highly reliable in the field $[8,9,15]$. As a low-cost alternative to tethered sensors, wireless sensors enable next-generation structural monitoring systems defined by high nodal densities. While wireless sensors have shown tremendous promise, their Achilles' heel remains power. To be more specific, battery powered wireless sensors can only operate for short periods before battery replacement is absolutely necessary. Urgently needed are viable power solutions that offer wireless sensors operational life spans on the order of decades without incurring additional cost or size.

This project will explore the design of an ultra low-power wireless sensor node for structural health monitoring. A conceptual diagram of the sensor node is shown in Fig. 4a. The single-chip sensing node will incorporate wireless transceiver, low power processor, capacitive and temperature sensors, and real time clock. Analog-to-digital converters will also be incorporated on the IC to record the voltage response of self-sensing materials electrically stimulated for EIT distributed sensing. In addition to being the primary data acquisition node for EIT sensing, traditional point sensors (e.g., accelerometers, strain gages, crack gages) can also be interfaced to the node's ADC for data collection. The onchip processor is designed to offer a powerful processing engine for on-board data interrogation. Individual elements including the radio, on-chip antenna and processor have already been demonstrated as proof-of-concept standalone devices implemented in mature $0.18 \mu \mathrm{m}$ CMOS technology. A key target in the node design is the minimization of the node's power consumption so that it can operate from scavenged energy stored in a compact chip-scale thin film battery. Hybrid power, from complimentary sources, offers the best possibility of meeting the differing power and energy requirements of the various modes of operation. While background processing and sensing can be sustained by energy scavenged from vibrations $(<100 \mathrm{nW})$, transient higher power levels $(<10 \mu \mathrm{W})$ required for intensive processing and data collection from sensors can be provided by a chip-scale battery intermittently re-charged by power harvesting devices. 


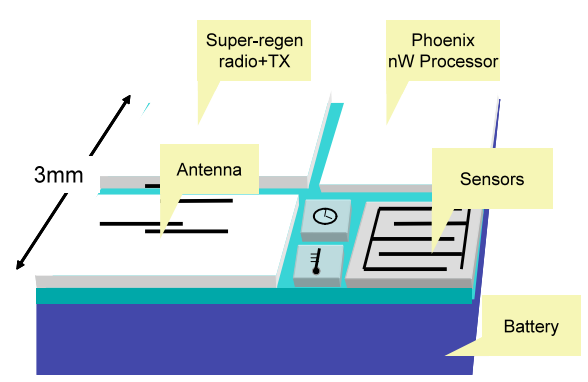

(a)

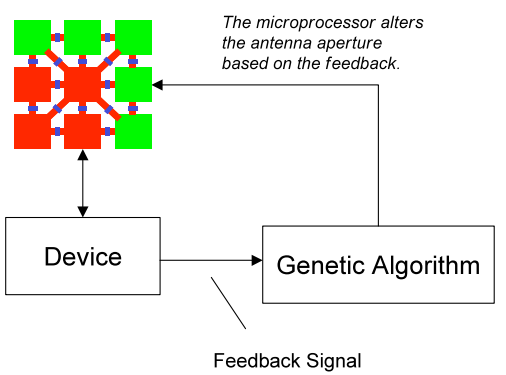

(b)

Fig. 4. (a) Ultra low-power wireless sensor node for infrastructure monitoring. (b) Cognitive antenna based on self-structuring antenna technology.

An additional challenge is the intermittent nature of scavenged energy, and therefore opportunistic techniques that exploit available energy will be explored. A promising approach to be investigated is an opportunistic analog-to-digital converter (ADC) that pauses and restarts a conversion cycle depending on the available energy.

Historically, wireless sensors for infrastructure monitoring have not considered the complex and challenging electromagnetic (EM) environment large structures pose. For example, steel reinforcement in concrete heavily shields wireless signals. Other challenges also include multipath effects and interference in operational infrastructure [16]. Poor performance of the wireless channel requires data to be transmitted repeatedly until it is delivered; repeated transmission unnecessarily consumes precious power. To minimize the effects of these detrimental phenomena, a selfstructuring antenna (SSA) is proposed to meet the rigorous demands of infrastructure EM environments. SSA dynamically alters the antenna aperture so as to maximize signal-to-noise ratios (SNR) in varying RF environments (see Fig. 4b) [17]. The proposed antenna aperture is made up of antenna elements connected via RF relays, which are turned on or off to form and maintain the optimum aperture for the perceived RF environment. A feedback from the radio provides a measure of signal quality for the optimizing algorithm, which in turn decides on the switch states. In effect, SSA offers adaptive frequency tuning and beam steering for wide-band as well as narrow-band applications. The advantages of adaptive beam-steering are evident when the EM environment is challenging including the existence of large physical obstacles between sensor nodes.

\subsection{Vibration and EM-based power harvesting}

A microelectromechanical system (MEMS) vibration power harvester will be developed to power the ultra low-power wireless sensor node. Environmental vibration is a particularly attractive energy source because of its abundance in infrastructure systems. However, the maximum electromotive force (emf) and corresponding generated electrical power from a vibrating mass are strongly dependent on the external vibration frequency with efficiencies dropping dramatically at low frequencies $(1-10 \mathrm{~Hz})$. Particularly for infrastructure systems that vibrate in this specific band, vibration-based harvesting has proven challenging for power devices like sensors [18]. In this effort, microelectromechanical system (MEMS) technology will be adopted to yield an electromagnetic vibration-to-electrical power generator optimized to scavenge energy from low-frequency external vibrations ( 1 to $100 \mathrm{~Hz}$ ). The operational concept of the device is based on the up-conversion of the inefficient low-frequency vibrations common in infrastructure to vibration frequencies better suited for efficient power harvesting. As shown in Fig. 5, a low-frequency resonator mass is used to actuate a smaller, high frequency resonator. The bigger resonator is suspended with a soft spring and has a low resonance frequency that is adjusted for the target application $(1-100 \mathrm{~Hz})$. The smaller resonator, which has a higher resonance frequency, supports a coil for electromagnetic power generation. Frequency up-conversion occurs when the low-frequency device collides with the high frequency device. By conservation of momentum, the large mass transfers its energy to the high frequency mass by setting it into a state of resonance. Analysis shows that such an up-converting scavenger can generate at least $20-50 \%$ more power than a typical low-frequency mechanical scavenger [19].

\subsection{In-network data processing in the wireless sensor network}

The cost of the proposed wireless sensor will be a fraction of that of existing sensors; in addition, the sensors will be free of battery sources. These attributes will usher in an era of dense sensing (i.e., hundreds, if not thousands of nodes within a single infrastructure system). However, the aforementioned energy constraints on wireless sensors make minimization 


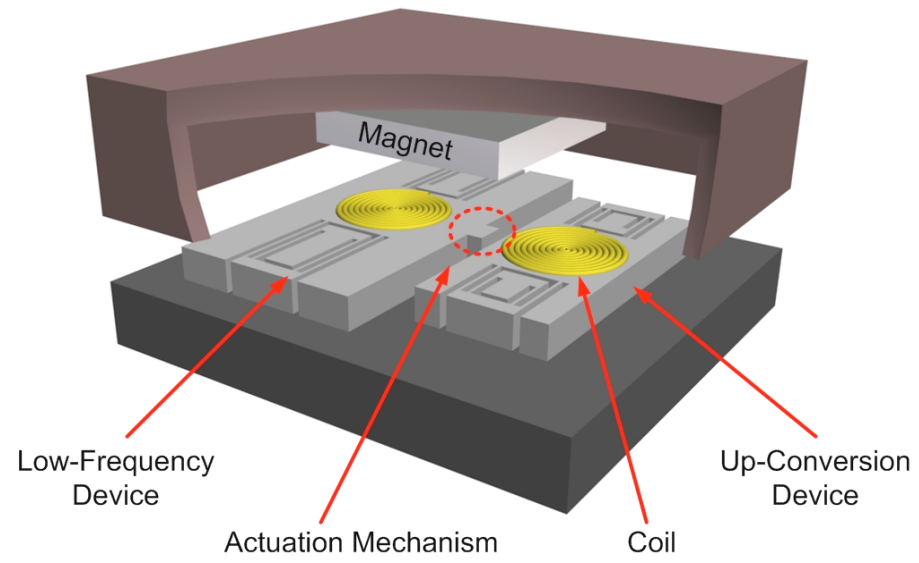

Fig. 5. Conceptual design of an "up-converting" vibration power scavenger.

of communication desirable since wireless communication consumes more power than any other sensor operation [9]. Significant power saving can therefore be gained by minimizing the amount of data to be transmitted. With computational resources (i.e., microcontroller) collocated with the sensor, sensor-based data interrogation can eliminate the need to continuously communicate raw time-history data. Sensor-based interrogation accomplishes other beneficial goals including: 1) the alleviation of burden on the wireless bandwidth by converting high bandwidth data streams (i.e., raw data) into low bandwidth streams, and 2) facilitation of scalable data management architectures for monitoring systems characterized by high nodal counts (as envisioned in this project). A variety of data processing algorithms will be explored for embedment in the wireless sensors including statistical analysis, modal analysis [20], model updating [21], and damage detection [22] algorithms. In particular, the full spectrum of computing architectures spanning from sensor-centric (decentralized) to network-centric (distributed) computing architectures will be considered to delicately balance computational performance against computational throughput, network reliability and overall network power efficiency.

\subsection{An SHM cyberenvironment}

Robust and secure multi-directional communication between wireless sensors installed on the bridge and data servers that will store and process relevant data must be established. It should be noted that the extremely high nodal densities will generate significant amounts of data to be transmitted to the central data servers that are remotely accessible via the internet. This problem can be addressed by a powered gateway node on the bridge that aggregates data from the various low-powered sensors and then periodically transmits the data over a wired or wide-area wireless (e.g., CDMA, Edge, or $3 \mathrm{G})$ network. To support the data management needs of the models and bridge response data, a neutral format database system will be designed to contain all structural information (e.g., geometry, stiffness, boundary conditions, loading), FEM models (regardless of the package) and measurement data of each structure. An owner's entire fleet of bridges can be managed within the same database. This supports statistical analysis of bridges constructed of the same type or located within the same regional area.

Once data has been aggregated in a set of servers on the internet, a rich multi-modal data set from multiple bridges is available for data mining. Specifically, bridge data must be analyzed to answer the following owner's questions: 1) is an infrastructure element in good health?; 2) do any underlying issues exist (e.g., under-designed elements or undetected damage) that can lead to sudden catastrophic failures?; 3) what is the most cost-effective maintenance and inspection strategy given current infrastructure conditions?; 4) what is the remaining life of an infrastructure element?; 5) what is the impact of management decisions on long-term economic, social and environmental sustainability indicators? Hence, a comprehensive analytical framework that will aggregate data flows from individual infrastructure elements will be developed to assist owners in answering these questions, thereby leading to more cost-effective management practices and avoidance of catastrophic failures such as bridge collapses. A brief description is provided for each computational element that will be developed in the project. 
Finite element modeling: finite element method (FEM) models are a standard analysis tool widely used during the design of bridge structures. While vital to understanding bridge response to design loads, FEM models are often stored after design but never validated nor updated. In this project, a computational framework is proposed that automates the task of maintaining FEM bridge models for use within bridge management processes. Data (raw or processed) derived from monitoring systems installed on infrastructure elements will be aggregated via the internet to be stored in large servers for sophisticated analysis using FEM models. The integration of response data with FEM models will be used to: 1) update FEM models in real-time so that bridge responses can be predicted in locations where no sensor are installed; 2) alert bridge managers when damage or deterioration occurs; and 3) provide inputs to life-cycle analyses and bridge management decision making.

System identification and model updating: In this study, global and local properties of instrumented bridges will be determined by system identification techniques [23]. For example, global modal properties (e.g., modal frequencies, mode shapes) will be estimated using bridge response data. Modal properties will be used to conduct model updating of existing FEM models. Local behavior can also be used to identify novel constitutive relationships that better represent the local behavior of key bridge elements.

Automated damage detection: Damage detection (DD) is the technique of determining if a change in the structure's design state has occurred [6]. Damage in a system is detectable if the outputs following the damage are statistically different from the outputs prior to damage. Damage isolation (DI) is the technique of differentiating between various damage states (e.g., fatigue cracking, corrosion, element yielding) that may occur in an infrastructure system. In this proposal, four major approaches to DD are proposed: fatigue modeling, pattern recognition, and innovations-based detection. For fatigue analysis of instrumented bridge components, classical rain flow counting will be adopted to continuously and autonomously count strain peaks measured in the system. Even for locations not instrumented, the cyclic response of all model elements in the updated FEM model will be tracked. Using peak amplitude strains (S) and the accumulated number of peaks $(\mathrm{N})$, Miner's rule and the appropriate S-N curve for steel will be used to roughly track the fatigue life of the structure and its critical elements [24]. Alternatively, a first order reliability method (FORM) approach will be adopted to identify remaining fatigue life in a structure's elements [25]. This probabilistic method is easy to implement and provides a reliability index which indirectly estimates the remaining life of a structural element. Finally, a generalized pattern recognition framework will also be developed to account for changes in a structure not necessarily attributable to fatigue cracking. Multiple approaches will be considered including Bayesian decision theory, support vector machines, and statistical clustering [26]. To employ pattern classification, feature vectors sensitive to damage will be devised. For example, time-series coefficients, modal properties, peak responses, extreme value statistical metrics can all serve as suitable features.

\subsection{Decision making tools}

While the value of providing hard facts to support decision-making is widely recognized, there is still a lack of support for using information effectively. Most traditional analysis systems available in the market are written for specific applications and can be cumbersome and un-intuitive to use. This leaves many struggling with mounds of valuable data but little information of timely value. Hence, an innovative new software solution known as a Decision Making Toolbox (DMT) is proposed (Fig. 6). DMT will transform raw and processed data obtained from the wireless monitoring system into relevant information for intelligent decision-making. DMT is envisioned as a coherent, comprehensive set of analysis programs that will not only be a seamless blend of tools, but also provide a medium to develop rapid application analysis without any programming through a "visual modeling" environment. All it takes will be a mouse to select library objects, arrange them on the screen and assign properties. This programming-free DMT will be a truly multidimensional environment that will include many of the analytical tools developed in this project: 1) high performance multidimensional database, capable of handling extremely large amounts of data, 2) tools for extraction/transformation/loading data, 3) a powerful yet simple front-end development environment (client and web) for building applications that range from simple reporting to complex analysis and simulation, and 4) security and a multitier architecture for system delivery.

The automatic synchronization and data-awareness of the plug \& play components will eliminate the need for high levels of technical expertise and substantially reduce the complexity and costs associated with building, customizing and maintaining applications. This approach allows one to create programming-free visual applications that effectively manage the response data stored in databases and to create analyses tailored to the decision making process. The available tools that fit the application architecture will be analyzed and integrated into DMT. This will enable the end 


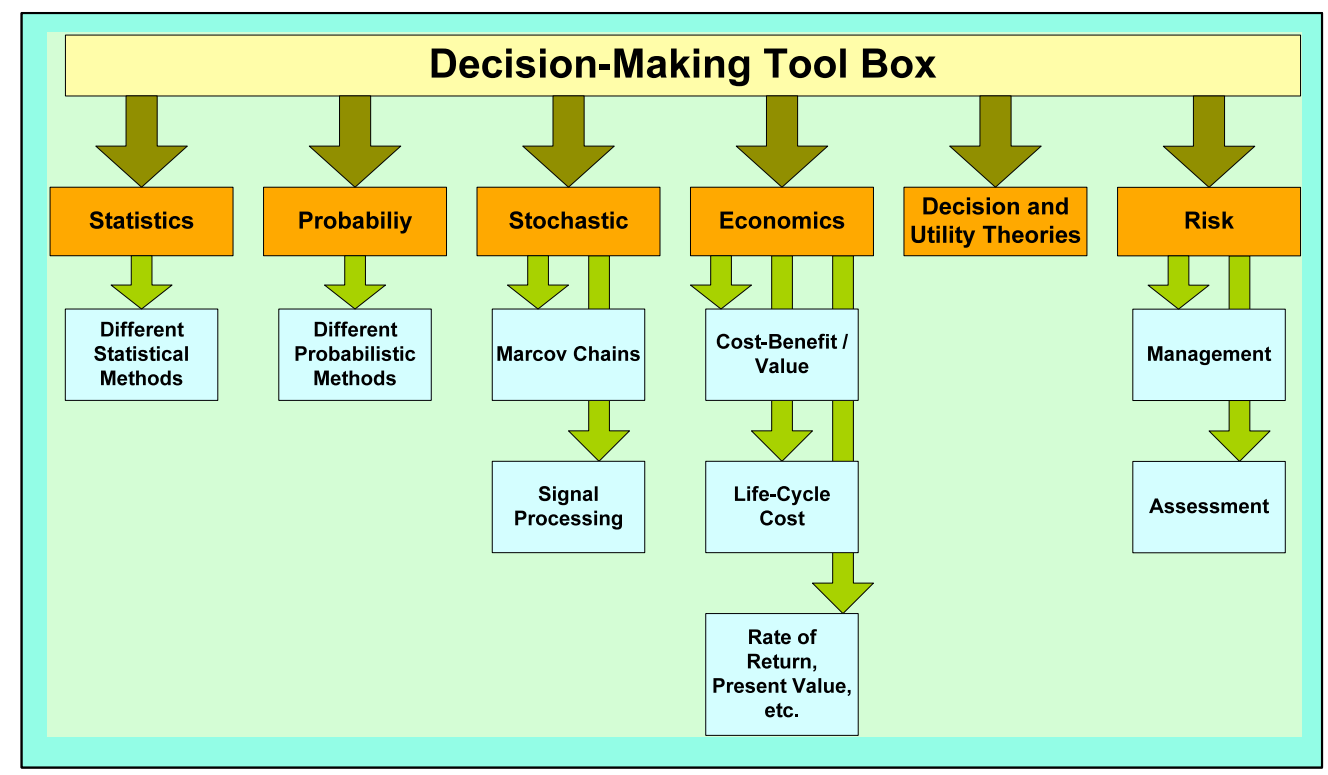

Fig. 6. Decision making toolbox concept.

users (i.e., bridge owners) to easily use the tools available in the DMT and if needed to integrate, at will, their own applications.

\subsection{Human interaction with sensed infrastructure}

The advances proposed in this project will not completely replace the need for human inspectors in the field. Rather, the proposed monitoring system is intended to influence the current state of practice by assisting asset managers with a means of prioritizing which bridges get inspected and when. Furthermore, sensors installed on the bridge structure will offer inspectors the opportunity to add greater objectivity to their inspections. To enhance the visual inspection process, wireless interaction between human inspectors and the pervasive sensor network installed in a bridge will be offered by the monitoring system. The specific goal is to design and implement a context-aware mobile computing [27] platform that will be capable of automatically identifying an inspector's spatial context, and retrieve bridge information and sensor data that is of relevance to the inspector's tasks at a particular time and location. The proposed approach is graphically depicted in Fig. 7.

In this study, the spatial context of an inspector will be automatically and continually deciphered by tracking two primary pieces of information: 1) the position where the inspector is located; and 2) the direction in which the inspector is looking. The platform will rely on the Global Positioning System (GPS) and three-dimension orientation trackers to monitor an inspector's real-time location and heading (i.e., direction of view). GPS will measure the user's position in the form of longitude, latitude, and altitude. The magnetic tracker will also measure the orientation of the user's head in the form of roll, pitch, and yaw. These 6 parameters (longitude, latitude, altitude, roll, pitch, and yaw) can fully define the user's location at any given time. Having this information, the inspector's line-of-sight can also be accurately calculated. Using these results, the region of space that is currently in the user's field of view will be derived using geometric results from computer graphics. In particular, the space in the user's view will be assumed to be a truncated pyramid (i.e., viewing frustum) whose corners can be geometrically calculated based on the user's location and line of sight. The obtained viewing frustum can then be geo-referenced (i.e., overlapped) with existing graphical CAD data of the bridge in the user's surroundings. The CAD objects lying inside the viewing frustum and intersecting with the user's line of sight are the bridge components that are in context at that particular time and location. This information can then be interactively presented to the engineer or inspector in real-time on a wirelessly-enabled heads-up display. In addition, the inspector will be able to wirelessly communicate with the bridge sensor network to retrieve data from the sensors and to access databases remotely located on the internet. 


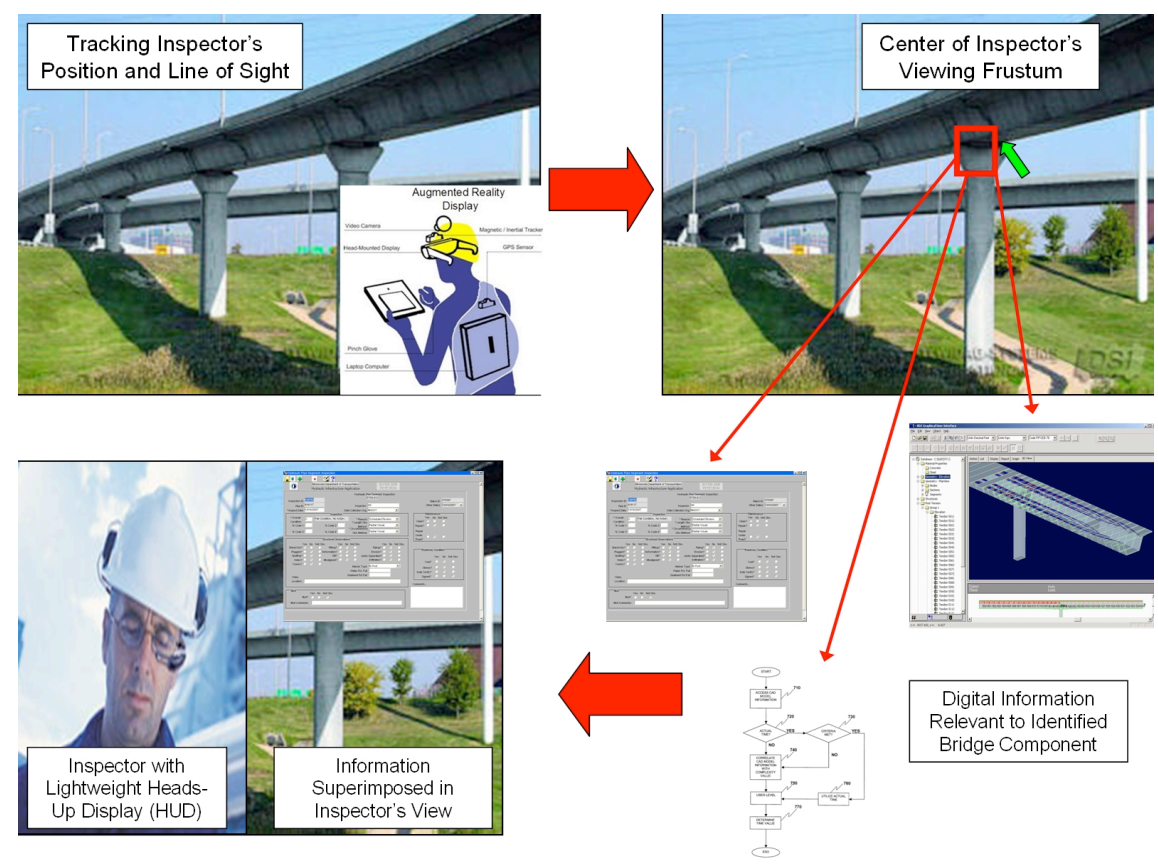

Fig. 7. Context-aware information access methodology.

\section{CONCLUSIONS}

A comprehensive structural health monitoring system is proposed for monitoring bridge infrastructure. The monitoring system offers innovations on multiple length-scales (i.e., at the component, structure, and regional length-scales) to overcome historical hurdles that limit the attractiveness of current structural health monitoring systems. Specifically, self-sensing engineered cementitious composites are proposed to offer distributed, direct sensing of cracking and deterioration in cementitious infrastructure components. To collect data from a vast number of electrical electrodes used to probe self-sensing ECC components, as well as from traditional sensors, an ultra low-power wireless sensor node is proposed. Vibration power harvesting devices are also explored for integration with the low-power wireless sensor node. Additional operational power efficiency are gained by using distributed computing architectures within the wireless sensor network itself. Data from sensors embedded in a bridge flows to the internet using internet access points or wide-area wireless networks. Bridge measurement data is then used to perform updating of finite element models and to drive damage detection algorithms searching for the onset of structural deterioration. Processed data is then forwarded to end-users in two ways. First, a decision making toolbox is used to aggregate data and to translate it into information a bridge owner needs to make effective decisions on future management of resources (e.g., repair budgets, inspection personnel). Second, field inspectors employing heads-up displays and wireless transceivers will be capable of fully interacting with bridge sensors and processed data. Plans are underway to validate the proposed cyber-enabled wireless structural health monitoring system on bridges in Michigan and California.

\section{ACKNOWLEDGMENTS}

The authors would like to gratefully acknowledge the generous support offered by the U.S. Department of Commerce, National Institute of Standards and Technology (NIST) Technology Innovation Program (TIP) under Cooperative Agreement Number 70NANB9H9008. Additional support was provided by the University of Michigan, Michigan Department of Transportation (MDOT) and the California Department of Transportation (Caltrans). The intellectual contributions offered by Prof. Mingyan Liu, Prof. Amir Mortazawi, Prof. Atul Prakash, Prof. Amit Ghosh, and Mr. Todd Anuskiewicz are greatly appreciated. 


\section{REFERENCES}

[1] Wardhana, K. and Hadipriono, F. C., "Analysis of recent bridge failures in the United States", Journal of Performance of Constructed Facilities, 17 (3), 144-150 (2003).

[2] Anderson, J., "Cities debate privatizing public infrastructure", The New York Times, New York, August 26 (2008).

[3] Rolander, D. D., Phares, B. M., Graybeal, B. A., Moore, M. E. and Washer, G. A., "Highway bridge inspection: State-of-the-practice survey", Transportation Research Record, 1749 (1), 73-81 (2001).

[4] Moore, M., Phares, B., Graybeal, B., Rolander, D. and Washer, G., [Reliability of visual inspection for highway bridges], Federal Highway Administration, Washington D. C. (2001).

[5] Adams, D., [Health monitoring of structural materials and components], Wiley, Hoboken, NJ (2007).

[6] Doebling, S. W., Farrar, C. R., Prime, M. B. , "Summary review of vibration-based damage identification methods", Shock and Vibration Digest, 30 (2), 91-105 (1998).

[7] Sohn, H., Farrar, C. R., Hemez, F. M., Shunk, D. D., Stinemates, S. W., Nadler, B. R. and Czarnecki, J. J., [Review of structural health monitoring literature from 1996-2001], Los Alamos National Laboratory, Los Alamos, NM (2004).

[8] Straser, E. and Kiremidjian, A. S., [Modular, wireless damage monitoring system for structures], John A. Blume Earthquake Engineering Center, Stanford, CA (1998).

[9] Lynch, J. P. and Loh, K., "A summary review of wireless sensors and sensor networks for structural health monitoring", Shock and Vibration Digest, 38 (2), 91-128 (2006).

[10] Li, V. C. and Kanda, T., "Engineered cementitious composites for structural applications", Journal of Materials in Civil Engineering, 10 (2), 66-69 (1998).

[11] Hou, T. C. and Lynch, J. P., "Monitoring strain in engineered cementitious composites using wireless sensors", Proceedings of the International Conference on Fracture (ICF XI) Turin, Italy (2005).

[12] Hou, T. C. and Lynch, J. P., "Tomographic imaging of crack damage in cementitious structural components", Proceedings of the 4th International Conference on Earthquake Engineering, Taipei, Taiwan (2006).

[13] Barber, D. and Brown, B. H., [Progress in electrical impedance tomography], SIAM Press, Philadelphia, PA (1990).

[14] Sohn, H., Farrrar, C. R., Hemez, F. M., Shunk, D. D., Stinemates, S. W., Nadler, B. R. and Czarnecki, J. J., [Review of structural health monitoring literature from 1996-2001], Los Alamos National Laboratory, Los Alamos, NM (2004).

[15] Spencer, B. F., Ruiz-Sandoval, M. E. and Kurata, N., "Smart sensing technology: Opportunities and challenges", Journal of Structural Control and Health Monitoring, 11 (4), 349-368 (2004).

[16] Mittag, L., "Magic in the air", Embedded Systems Programming, 14 (10), $49-60$ (2001).

[17] Coleman, C. M., Rothwell, E. J., Ross, J. E. and Nagy, L. L., "Self-structuring antennas", IEEE Antennas Propagation Magazine, 44 (3), 11-22 (2002).

[18] Ashebo, D. B., Tan, C. A., Wang, J. and Li, G., "Feasibility of energy harvesting for powering wireless sensors in transportation infrastructure applications", Proceedings of SPIE: Nondestructive Characterization for Composite Materials, Aerospace Engineering, Civil Infrastructure, and Homeland Security, 69340Y, (2008).

[19] Kulah, H. and Najafi, K., "An electromagnetic micro power generator for low-frequency environmental vibrations", Proceedings of the 17th IEEE International Conference on Microelectromechanical Systems (MEMS), 237-240, Maastricht, Netherlands (2004).

[20] Zimmerman, A., Swartz, R. A., Saftner, D. A., Lynch, J. P., Shiraishi, M. and Setareh, M., "Parallel data processing architectures for identification of structural modal properties using dense wireless sensor networks", Proceedings of the World Forum on Smart Materials and Smart Structures Technology, Chongqing, China (2007).

[21] Zimmerman, A. T. and Lynch, J. P., "Distributed model updating in wireless sensor networks by simulated annealing", IEEE Sensors, in press (2009).

[22] Lynch, J. P., Sundararajan, A., Law, K. H., Kiremidjian, A. S. and Carryer, E., "Embedding damage detection algorithms in a wireless sensing unit for operational power efficiency", Smart Materials and Structures, 13 (4), 800810 (2004).

[23] Ljung, L. and Soderstrom, T., [Theory and practice of recursive identification], MIT Press, Cambridge, MA (1983).

[24] Brooks, C. R. and Choudhury, A., [Failure analysis of engineering materials], McGraw-Hill, New York, NY (2002).

[25] Frangopol, D. M., Strauss, A. and Kim, S., "Bridge reliability assessment based on monitoring", Journal of Bridge Engineering, 13 (3), 258-270 (2008).

[26] Duda, R. O., Hart, P. E. and Stork, D. G., [Pattern classification], Wiley, New York, NY (2001). 
[27] Burrell, J. and Gay, K., "Collectively defining context in a mobile, networked computing environment", ComputerHuman Interaction 2001, (2001). 